\title{
Patología implanto-endodóncica: Concepto, tipos, diagnóstico, tratamiento y prevención
}

\section{Endodontic-implant pathology: Concept, types, diagnosis, treatment and prevention}

\author{
RODRÍGUEZ-ORTEGA $L$ * \\ VELASCO-ORTEGA V* \\ CASTELLANOS-COSANO L** \\ MARTÍN-GONZÁLEZ L** \\ LÓPEZ-FRÍAS FJ** \\ SEGURA-EGEA JJ**
}

\begin{abstract}
Rodríguez-Ortega L,Velasco-Ortega E, Castellanos-Cosano L, Martín-González J, López-Frías FJ, Segura Egea JJ. Patología implantoendodóncica: Concepto, tipos, diagnóstico, tratamiento y prevención. Av Periodon Implantol. 2013; 25, 2: 83-90.
\end{abstract}

\section{RESUMEN}

La patología implanto-endodóncica (PIE) está descrita en la literatura implantológica como una de las causas de periimplantitis apical, entendida como la lesión osteolítica en la región apical del implante, con normal osteointegración de su porción coronal, provocada por la infección por contigüidad a partir de la lesión periapical del diente adyacente. Pero el concepto de PIE no sólo abarca la periimplantitis retrógrada por contaminación diente-a-implante, sino también los procesos inflamatorios periapicales en dientes adyacentes al implante por contaminación implante-adiente, cuando la colocación del implante provoca la necrosis del diente adyacente y la consiguiente periodontitis apical. Incluso podríamos incluir dentro de la PIE los casos de periimplantitis apical en implantes postextracción provocada por la infección residual presente en el alvéolo de un diente extraído con periodontitis apical. En definitiva, la PIE incluye las lesiones endodóncicas e implantarias apicales que son el resultado de infecciones residuales o por contigüidad entre diente e implante. En esta revisión bibliográfica se define y clasifica la PIE, repasándose la casuística publicada así como su influencia en el resultado del tratamiento implantológico.

PALABRAS CLAVE: Enfermedad endodóncica, implante postextracción, patología implanto-endodóncica, periimplantitis apical, periimplantitis retrógrada, periodontitis apical.

\section{SUMMMARY}

The implant-endodontic pathology is described in the literature as a cause of apical periimplantitis, understood to be an osteolytic lesion that appears in the apical region of the implant during the coronal osseointegration normally. This implant-endodontic pathology researches the relation of endodontic and implants apical lesions leading to infection contiguity between teeth and implants. The first way of contamination is implant-tooth, when the implant placement causes necrosis of the adjacent tooth and subsequent contamination of the implant; the second way is tooth-implant, when there is an exacerbation of latent apical lesion in a tooth, root canal or not, after placing an implant adjacent to it, resulting in apical periimplantitis later.

In this work we have summoned the cases of occurring periimplantitis by apical residual infection present in the socket of an extracted tooth with apical periodontal pathology and replaced by an implant. This review aims to make an update of the relationship between periimplantitis and endodontics. 
KEYWORDS: Apical periimplantitis, apical periodontitis, endodontic disease, implant-endodontic pathology, retrograde periimplantitis.

Fecha de recepción: 10 de julio 2011 .

Fecha de aceptación: 10 de septiembre 2012.

\section{INTRODUCCIÓN}

En los últimos años, el foco de la literatura implantológica ha ido cambiando progresivamente de la descripción de las tasas de éxito de los implantes hacia el estudio detallado de sus complicaciones y fracasos. Una de estas complicaciones es la periimplantitis apical o retrógrada, una de las posibles causas de fracaso implantológico (1). La periimplantitis retrógrada se manifiesta como una lesión osteolítica que aparece en la región apical de los implantes dentales, mientras la porción coronal del implante sigue osteointegrada normalmente. La periimplantitis retrógrada o apical puede presentarse en dos formas, inactiva y activa (2). La presencia de una radiolucidez apical asintomática indicará la forma inactiva de esta lesión. Si, además, se acompaña de síntomas inflamatorios como dolor y/o supuración, nos encontraremos ante una forma activa de periimplantitis retrógrada. Esta lesión puede desarrollarse en estadios tempranos tras la inserción de los implantes $(3,4)$, o incluso años después (5).

Las causas de la periimplantitis apical son diversas, pudiendo agruparse en tres tipos de factores: 1) los dependientes del propio paciente, como patologías preexistentes, como un seno maxilar contaminado, una periodontitis apical en un diente vital adyacente, o un foco inflamatorio crónico en un diente endodonciado contiguo o en un alvéolo postextracción $(6,7)$, la presencia de restos radiculares (8) o cuerpos extraños en el hueso, el tabaco (9) o hueso de pobre calidad (10); 2) los dependientes del propio implante, como su diseño, su superficie (11) o la contaminación del mismo (7); y 3) los dependientes del operador y del protocolo terapéutico, como el sobrecalentamiento del hueso en la colocación del implante $(12,13)$, microfracturas por sobrecarga, carga temprana o fuerzas laterales (14), cavidades óseas residuales por la colocación de implantes de longitud menor al lecho preparado (15), implantación accidental de células epiteliales gingivales en el lecho quirúrgico (16) o no respetar una distancia adecuada con los dientes adyacentes (2 mm) (17).

Por otra parte, la periodontitis apical en los dientes naturales es el resultado de la reacción inflamatoria provocada por los antígenos procedentes de los conductos radiculares necróticos e infectados (18). Cuando esta infección continúa y se cronifica tras el tratamiento de conductos, se convierte en una periodontitis apical crónica persistente (PACP). Las causas de la PACP tras el tratamiento endodóncico no han sido bien aclaradas, y siguiendo la literatura, pueden dividirse en microbianas y no microbianas. Dentro de las causas microbianas, la PACP puede ser secundaria a la persistencia de biofilms bacterianos en el conducto radicular ya obturado (19), al no haberse conseguido la completa remoción bacteriana. También puede ser consecuencia de una infección extrarradicular, habiéndose descrito la presencia extrarradicular de actinomices y otros microorganismos extracelulares (20), así como diferentes formas víricas latentes que son activadas por la inflamación periodontal (21). Entre las causas no microbianas de PCAP se encuentran los quistes apicales (22), los cristales de colesterol, posibles cuerpos extraños transportados al periápice durante el tratamiento de conductos o la presencia de tejido reparativo en la zona de resolución de la lesión (23). En los últimos años se han descrito casos de necrosis pulpar y periodontitis apical en dientes adyacentes a implantes recién insertados (24).

\section{PATOLOGÍA IMPLANTO- ENDODÓNCICA}

La posibilidad de infecciones por contigüidad entre el implante y el diente adyacente ha dado origen al concepto de "patología implanto-endodóncica". En 1998, Sussman y cols. (24) acuñaron el término "Patología implanto-endodóncica" (endodontic implant pathology) a partir de los conceptos de periimplantitis retrógrada y periodontitis apical, clasificándola según su etiología en dos grupos:

1. PIE tipo 1, implante-a-diente, "implant endodontitis" según Sussman y cols. (24), cuando tras la inserción del implante (lesión implante-a-diente), se produce la necrosis y periodontitis apical aguda del diente vital adyacente o la reactivación de un foco infeccioso periapical en el diente endodonciado 
contiguo, con la consiguiente aparición de signos clínicos y radiológicos de periodontitis apical.

2. PIE tipo 2, diente-a-implante, "endodontic implantitis" según Sussman y cols. (24), entendida como la lesión osteolítica en la región apical del implante, con normal osteointegración de su porción coronal, provocada por la infección por contigüidad a partir de la lesión periapical del diente adyacente.

Aunque Sussman y cols. (24) no la incluyen, pensamos que también podría incluirse dentro de la PIE los casos de periimplantitis apical en implantes postextracción provocada por la infección residual, de origen endodóncico, presente en el alvéolo de un diente extraído con periodontitis apical. Se trataría de la PIE tipo 3 , con infección alvéolo-a-implante.

En definitiva, la PIE incluiría las lesiones endodóncicas e implantarias apicales resultado de infecciones residuales o por contigüidad entre diente e implante. En esta revisión bibliográfica se define y clasifica la PIE, repasándose la casuística publicada así como su influencia en el resultado del tratamiento implantológico.

\section{METODOLOGÍA}

Se ha realizado una búsqueda bibliográfica en las bases de datos Pubmed y Scielo. Se han utilizado como palabras clave las siguientes: perimplantitis[All Fields]; endodontic[All Fields] AND pathosis[All Fields]; periodontitis" [MeSH Terms] OR ("periapical" [All Fields] AND "periodontitis" [All Fields]) OR "periapical periodontitis"[All Fields] OR ("apical"[All Fields] AND "periodontitis" [All Fields]) OR "apical periodontitis" [All Fields]; retrograde[All Fields] AND ("periimplantitis" [MeSH Terms] OR "peri-implantitis" [All Fields] OR "periimplantitis"[All Fields]).

\section{PATOLOGÍA IMPLANTO- ENDODÓNCICA: INFECCIÓN POR CONTIGÜIDAD IMPLANTE-DIENTE}

En 1973, Peppersak y cols. (25) y, posteriormente, Banks y cols. (26), observan el desarrollo de necrosis pulpar en los incisivos inferiores tras osteotomías realizadas en la premaxila, relacionando este hecho con el sobrecalentamiento óseo y el pequeño diámetro apical de los dientes inferiores, más susceptibles de sufrir necrosis. Posteriormente, Sussman y cols. $(24,27)$ exponen el concepto de patología implanto-endodóncica, y Margelos y cols. (28) presentan 3 casos clínicos en los que asocia la colocación de implantes y el desarrollo de pulpitis irreversible en los dientes adyacentes, con el posterior fracaso de los implantes. Piatelli y cols. (29) plantean la posibilidad de que la periodontitis apical crónica pueda dar lugar al fracaso de los implantes adyacentes y ese mismo año Sussman y cols. (24) clasifican las infecciones por contigüidad entre diente e implante y analizan su patogenia.

La PIE tipo 1, implante-a-diente, se produce cuando la inserción del implante provoca la necrosis pulpar y la periodontitis apical del diente adyacente previamente sano. Estaría provocada por el trauma directo durante la preparación del lecho implantario (por invasión del espacio periodontal, sobrecalentamiento del hueso o interrupción del aporte sanguíneo a la pulpa) o por infección intraoperatoria. La lesión endodóncica que se produce se manifiesta por una lesión osteolítica periapical, radiolúcida, que puede afectar por contigüidad al implante y alterar su osteointegración.

La PIE tipo 2, diente-a-implante, se desarrolla cuando tras la colocación del implante se produce la exacerbación de un proceso infeccioso/inflamatorio periapical previo del diente adyacente, endodonciado (PIE tipo 2-A) o no endodonciado (PIE tipo 2-B), que afecta por contigüidad al implante. Se evidencia por la aparición de una lesión radiolúcida en la región periapical del implante que se extiende desde el diente adyacente, desarrollada poco después de la inserción, acompañada de dolor, sensibilidad, hinchazón y presencia de un trayecto fistuloso (24).

Por último, la PIE tipo 3, alvéolo-a-implante, no sería mas que la infección de la porción apical del implante tras su inserción en un alvéolo infectado como consecuencia de la patología endodóncica del diente al que sustituye (5).

\section{INFLUENCIA DE LA PATOLOGÍA IMPLANTO-ENDODÓNCICA EN EL ÉXITO DEL TRATAIMIENTO IMPLANTOLÓGICO}

Laird y cols. (30) estudiaron en 116 pacientes a los que se colocaron 233 implantes, la supervivencia de implantes y dientes adyacentes, endodonciados o no. Realizaron tres grupos:

A) Implantes sin dientes adyacentes.

B) Implantes con dientes adyacentes no tratados endodóncicamente.

C) Implantes con dientes adyacentes endodonciados. 
Los autores no encuentran diferencia significativa en la tasa de éxito encontrada en los tres grupos. Al igual que Shabahang y cols. (31), concluyen que la colocación de implantes al lado de dientes endodonciados es predecible y estos dientes deben ser mantenidos en boca.

Zhou y cols. (17) estudiaron la incidencia de periimplantitis apical en 128 implantes adyacentes a dientes con o sin tratamiento de conductos. Esta incidencia fue mayor en aquellos que estaban contiguos a dientes endodonciados, pero en el $70 \%$ de los casos los implantes se habían colocado a menos de $2 \mathrm{~mm}$ de los dientes adyacentes y las endodoncias se habían realizado hacia menos de 4 semanas de la colocación de los implantes.

Quirynen y cols. (32) realizan un estudio retrospectivo sobre 359 implantes, observando más casos de periimplantitis retrógrada en lugares donde se habían colocado implantes en reposición de dientes extraídos con patología apical o adyacentes a dientes con patología endodóncica obvia.

Por el contrario, Siegenthaler y cols. (33), en un estudio sobre 34 implantes, relacionan el éxito implantológico con la estabilidad primaria conseguida, sin encontrar diferencias significativas entre implantes postextracción en lugares con o sin patología periapical previa.

En un trabajo similar, realizado por Linderboom y cols. (34), tras colocar 25 implantes inmediatos, y 25 implantes a los 3 meses de las extracciones dentarias, encuentran a los 6 meses una supervivencia del $92 \%$ de los implantes en el primer grupo, y del $100 \%$ en el segundo, sin haber diferencias significativas en cuanto a valor ISQ.

También Crespi y cols. (35) estudian el éxito de implantes postextracción en zonas con o sin patología periapical previa, sin encontrar diferencias significativas en cuanto a salud periimplantaria y pérdida ósea.

Chang y cols. (36) comparan, en un estudio sobre perros, la osteointegración obtenida con implantes inmediatos en zonas libres de infección y zonas en las que se induce la patología perirradicular y se realiza la apicectomía de las piezas dentales a reponer con implantes, con colocación de membrana en uno grupo y sin ella en el otro. A las 12 semanas de la colocación de los implantes, en el grupo control hubo significativamente $>$ contacto hueso-implante que en los grupos experimentales: $1^{\circ}$ grupo y $2^{\circ}$ grupo, considerando predecible, pese a los resultados, la colocación de implantes inmediatos en lugares con previa patología periapical.

\section{INFLUENCIA DE LA PATOLOGÍA IMPLANTO-ENDODÓNCICA EN EL ESTADO PULPO-PERIAPICAL DEL DIENTE ADYACENTE}

Misch y cols. (37), en un estudio retrospectivo sobre 1.277 implantes adyacentes a 2.589 dientes, observaron que, en 10 años de seguimiento, sólo el 0,4\% requirió endodoncia y ningún diente fue extraído. Concluyen que, si la posición de los implantes es adecuada, respetando una distancia suficiente con los dientes adyacentes, estos últimos no tienen por qué verse afectados endodóncicamente.

\section{DIAGNÓSTICO DE LA PATOLOGÍA IMPLANTO-ENDODÓNCICA}

La causa originaria de la lesión, endodóncica o implantológica es de difícil diagnóstico cuando la imagen radiolúcida abarca el ápice implantario y de dientes adyacentes. Las pruebas de vitalidad y percusión, así como el estado de los tratamientos endodóncicos en los dientes adyacentes, nos alertarán de la posible degeneración pulpar y presencia de patologías perirradiculares.

La comprobación de la adecuada posición de los implantes colocados, a una distancia apropiada de los dientes vecinos, así como la estabilidad de los mismos, corroborando que no haya un fracaso en la osteointegración, nos ayudarán a realizar un buen diagnóstico de la periimplantitis apical (17).

En el año 2001, Brisman y cols. (38) publican un polémico artículo en el que comunican la aparición de perimplantitis retrógrada en 4 casos clínicos en los que los implantes se habían colocado adyacentes a dientes endodonciados y sin patología periapical. Tras estas observaciones concluyen que los dientes endodonciados siempre presentan bacterias remanentes en la zona periapical, aunque no tengan sintomatología clínica ni signos radiológicos, proponiendo el retratamiento endodóncico, la apicectomía o la extracción de forma sistemática de los dientes que van a ser adyacentes a futuros implantes. Este trabajo tuvo muchas críticas $(39,40)$ pues, de los 4 casos presentados, la aproximación excesiva de los implantes a los dientes adyacentes era evidente en dos de ellos, mientras que en los otros dos la relación entre el fracaso endodóncico y la periimplantitis retrógrada consiguiente no tenía solidez. 


\section{TRATAMIENTO DE LA PATOLOGÍA IMPLANTO-ENDODÓNCICA}

\author{
Tratamiento de la patología implanto- \\ endodóncica tipo 1 (implante-a-diente)
}

Hay pocos casos en la literatura en los que se describan lesiones periapicales en dientes adyacentes a implantes. Margelos y cols. (28) y, posteriormente, Sussman y cols. (24) presentan varios casos de periodontitis apical consecutiva a la colocación de un implante en los que la lesión endodóncica se resolvió realizando el tratamiento de conductos del diente que se había necrosado. En cambio, hubo que extraer los implantes debido a la lesión.

\section{Tratamiento de la patología implanto- endodóncica tipo 2 (diente-a-implante)}

Por el contrario, son numerosos los casos publicados de periimplantitis retrógrada secundaria a la infección del diente adyacente. Cuando el diente no está endodonciado (PIE tipo 2-B), Steiner y cols. (41) presentan dos casos de periimplantitis apical en los que la realización del tratamiento de conductos en el diente afectado resuelve la lesión endodóncica e implantológica, demostrando que la patología endodóncica en dientes adyacentes a implantes no siempre causa fracaso implantológico. Tozum y cols. (2) comunican un caso similar en el que se completa el tratamiento con la regeneración ósea guiada de la zona.

Por lo que a casos de PIE tipo 2-A se refiere, en los que el diente adyacente estaba endodonciado, Tae-Jou y cols. (42) y Tseng y cols. (43) presentan casos clínicos en los que, tras desarrollarse periimplantitis apical por exacerbación de la lesión periapical de los dientes adyacentes endodonciados, los casos se resuelven realizando la extracción del implante, la apicectomía del diente endodonciado y la regeneración ósea guiada de la zona, colocando nuevos implantes a los 3 meses, en el primer caso, y a los 6 meses en el segundo.

\section{Tratamiento de la patología implanto- endodóncica tipo 3 (alvéolo-implante)}

Respecto a la periimplantitis retrógrada provocada por la infección residual del alvéolo tras la extracción, Roshendal y cols. (5) muestran dos casos de periimplantitis apical en implantes que se habían colocado en el lugar de incisivos centrales superiores, extraídos por fracaso endodóncico y PACP. En ambos se realizó la resección apical del implante con buenos resultados.

Por último, sea cual sea la causa de aparición de la periimplantitis apical, el tratamiento debe completarse con medicación antibiótica $(1,2,5,9,44)$. Romanos y cols. (45), recomiendan además realizar un abordaje quirúrgico de la lesión en todos los casos, que puede abarcar desde el desbridamiento de la lesión, detoxificación de la superficie del implante o resección del ápice implantológico, a la regeneración ósea guiada de la zona. Balshi y cols. (46) realizan un estudio sobre 39 casos de periimplantitis apical, con 4,5 años de seguimiento, en el que consiguen un éxito del $97,4 \%$ incluyendo siempre la resección apical del implante en el tratamiento quirúrgico.

\section{PREVENCIÓN DE LA PATOLOGÍA IMPLANTO-ENDODÓNCICA}

Respecto a la prevención de la PIE tipo 1, los autores destacan la importancia de respetar una distancia adecuada diente-implante así como una correcta dirección en la colocación del implante que no produzca la interrupción del aporte vascular de los dientes vitales adyacentes (28). Consideran, además, que evitar el sobrecalentamiento en la osteotomía es básico para no necrosar los dientes adyacentes, con especial cuidado en la premaxila, de mayor densidad ósea (47).

Para prevenir la PIE tipo 2 en los casos en los que el diente adyacente no está endodonciado, se deben realizar pruebas de vitalidad pulpar en dientes adyacentes sospechosos de necrosis pulpar (como en aquellos que hayan sufrido algún traumatismo), así como realizar la endodoncia intencionada antes de la colocación de los implantes en los casos en los que haya grandes caries o fracturas cercanas a la pulpa (48).

En relación con la prevención de la PIE tipo 2 cuando el diente contiguo está endodonciado, Zhou y cols. (17), en su estudio sobre periimplantitis retrógrada en implantes adyacentes a dientes endodonciados, concluyen que la incidencia de periimplantitis retrógrada se puede ver reducida aumentando la distancia entre el implante y el diente vecino y/o el tiempo transcurrido desde el tratamiento de conductos y la colocación del implante adyacente. Por su parte, Tae-Ju Oh y cols. (42) recomiendan realizar el retratamiento endodóncico de los dientes adyacentes a implantes cuando existan dudas sobre la existencia de periodontitis apical crónica persistente (paciente asintomático con imagen radiolúcida periapical), evitando así la contamina- 
ción del implante. Tseng y cols. (43) aconsejan realizar la apicectomía del diente endodonciado adyacente con PACP en el mismo acto quirúrgico de inserción de los implantes. Por otra parte, Mohamed y cols. (48) recomiendan evitar el sobrecalentamiento óseo en la preparación del lecho del implante, ya que puede provocar la activación de posibles lesiones periapicales latentes en los dientes adyacentes endodonciados.

Por último, en los casos en los que las extracciones de los dientes a reponer con implantes se deban a fracasos endodóncicos, se debe prevenir la PIE tipo 3 mediante la administración de antibióticos bactericidas como la amoxicilina, clindamicina y eritromicina (estos 2 últimos son bacteriostáticos o bactericidas dependiendo de la concentración), mejor que con bacteriostáticos como la doxiciclina (9). Novaes y cols. (49) estudian el éxito de los implantes inmediatos en zonas con patología periapical preexistente. Presentan 3 casos clínicos en los que realizan premedicación antibiótica, y el desbridamiento, irrigación y regeneración ósea el día de la colocación de los implantes inmediatos, con buenos resultados a 3 años de seguimiento. Casap y cols. (50) nos proponen un tratamiento similar en un estudio sobre 30 implantes colocados en 20 pacientes con un éxito del $97 \%$, contraindicando los implantes inmediatos si el día de la cirugía la infección continúa activa tras la premedicación antibiótica. No obstante, Silva y cols. (51) no consideran estas patologías activas como una contraindicación en la colocación de implantes postextracción. Naves y cols. (52) realizan implantes inmediatos en zonas con patología apical realizando una primera cirugía, antes de la extracción dental, para eliminar todo el tejido granulomatoso alrededor del ápice dentario, colocando hueso bovino y membrana. Abou Rass y cols. (53) realizan, además, en esta primera cirugía, la endodoncia y/o apicectomía en los dientes que sufran lesiones periapicales antes de su extracción. Con estos procedimientos se obtienen las ventajas de los implantes inmediatos, preservando la altura alveolar, conservando las papilas y eliminando la infección residual.

\section{CONCLUSIONES}

La realización de un buen diagnóstico y plan de tratamiento son esenciales para evitar la patología implanto-endodóncica, tanto la periimplantitis apical retrógrada de origen endodóncico como la periodontitis apical de origen implantario. La periodontitis apical de origen implantario se puede prevenir respetando una distancia adecuada entre el implante y los dientes adyacentes, y evitando el sobrecalentamiento óseo que puede afectar a los tejidos periapicales y al tejido pulpar del tercio apical radicular. La periimplantitis apical, por infección por contigüidad a partir del diente adyacente al implante, puede evitarse comprobando la vitalidad de los dientes vecinos y la ausencia de patología periapical antes de la colocación del implante, así como verificando el correcto tratamiento de conductos de los dientes desvitalizados, respetando una distancia correcta entre diente-implante. La periimplantitis apical, desarrollada a partir de la infección residual del alvéolo del diente a sustituir por un implante, se previene prescribiendo premedicación antibiótica y realizando un correcto legrado. En todos los casos, se realizarán controles radiográficos que permitan diagnosticar los primeros estadios del desarrollo de una periimplantitis apical o una periodontitis apical secundaria a la colocación del implante. Los estudios de éxito/fracaso de implantes adyacentes a dientes endodonciados concluyen que no existe diferencia en las tasas de éxito y supervivencia de dichos implantes comparadas a las de implantes sin dientes endodonciados adyacentes. No existe evidencia científica que apoye la exodoncia preventiva de dientes adyacentes a los lechos implantarios como medida de prevención para evitar la periimplantitis retrógrada.

\section{BIBLIOGRAFÍA}

1. Ayangco L, Sheridan PJ. Development and treatment of retrograde peri-implantitis involving a site with a history of failed endodontic and apicoectomy procedures: a series of reports. Int J Oral Maxillofac Implants 2001; 16:412-7.

2. Tozum T, Sencimen M, Ortakoglu K, Ozdemir A, Aydin $\mathrm{O}$, Keles M. Diagnosis and treatment of a large periapical implant lesion associated with adjacent natural tooth: a case report. Oral Surg Oral Med Oral Pathol Oral Radiol Endod 2006;101:e132-e8.

3. Peñarrocha M, Boronat-López A, García-Mira B. Inflammatory implant periapical lesion: etiology, diagnosis, and treatment. Presentation of 7 cases. J Oral Maxillofac Surg 2009;67:168-73.

4. Chang LCh, Hsu ChS, Lee Yl. Successful medical treatment of an implant periapical lesion: a case report. Chang Gung Med J. 201 1;34:109-14.

5. Rosendhal K, Dahlberg G, Kisch J, Nilner K. Implant periapical lesion. A case series report. Swed Dent J. 2009; 33:49-58. 
6. Siegenthaler D, Jung R, Hoderegger C, Roos M, Hammerle Ch. Replacement of teeth exhibiting periapical pathology by inmediate implants. A prospective, controlled clinical trial. Clin. Oral Impl. Res. 2007;18: 727-37.

7. Piattelli A, Scarano A, Balleri P, Favero GA. Clinical and histologic evaluation of an active "implant periapical lesión". A case report. Int J Oral Maxillofac Implants 1998;13:713-16.

8. Park SH, Sorensen WP, Wang HL. Management and prevention of retrograde peri-implant infection from retained root tips: two case reports. Int J Period Restor Dent. 2004;24:422-33.

9. Flannagan D. Apical (retrograde) peri-implantitis: acase report of an active lesion. J Oral Implant 2002;28: 92-6.

10. Scarano A, Di Domizio P, Petrone G, Iezzi G, Piattelli A. Implant periapical lesion: a clinical and histologic case report. J Oral Implantol 2000;26:109-13.

11. Quirynen M, Vogels R, Alsaadi G, Naert I, Jacobs R, van Steenberghe D. Predisposing conditions for retrograde peri- implantitis, and treatment suggestions. Clin. Oral Impl Res 2005;16:599-608.

12. Watanabe F., Tawada Y., Komatsu S., Hata Y. Heat distribution in bone during preparation of implant sites: Heat analysis by real-time thermography. Int J Oral Maxillofac Implants 1992;7:212-19.

13. Tehemar S. Factors Affecting heat generation during implant site preparation: a review of biologic observations and future considerations. Int J Oral Maxillofac Implants 1999;14:127-36.

14. Meffert RM. Periodontitis and peri-implantitis: one and the same. Pract Periodontics Aesthet Dent 1993;5:79-80.

15. Reiser GM., Nevins M. The implant periapical lesion: Etiology, prevention and treatment. Compend Contin Educ Dent 1995;16:768-77.

16. Scarano A, Di Domizio P, Petrone G, Iezzi G, Piattelli A. Implant periapical lesion: a clinical and histologic case report. J Oral Implantol 2000;26:109-13.

17. Zhou W, Han Ch, Li D, Li Y, Song Y, Z Y. Endodontic treatment of teeth induces retrograde peri- implantitis. Clin Oral impl Res 2009;20:1326-32.
18. Siqueira JF. Endodontic infections: concepts, paradigms, and perspectives. Oral Surg Oral Med Oral Pathol Oral Radiol Endod 2002;94:281-93.

19. Sunqvist G, Reuterving CO. Isolation of actinomyces israelii from periapical lesion. J Endod 1998;6:602-6.

20. Figdor D, Davies JK, Sundqvist G. Starvation survival, growth and recovery of enterococus faecalis in human serum. Oral Microbiology and Inmunology 2003;18: 234-9.

21. Sabeti M, Slots J. Herpesviral-bacterial coinfection in periapical pathosis. J Endod 2004; 30:69-72.

22. Nair P.N.R, Pajarola G, Schroeder HE. Types and incidence of human periapical lesions obtained with extracted teeth. Oral Surg Oral Med Oral Pathol 1996;81: 93-102.

23. Nair P.N.R.Sjogren U, Figdor D, Sundqvist G. Persistent periapical radiolucencies of root filled human teeth, failed endodontic treatments and periapical scars. Oral Surg Oral Med Oral Pathol 1999;87:617-27.

24. Sussman H., Moss S. Periapical implant pathology.J Oral Implantol 1998;24:133-8.

25. Pepersack WJ. Tooth vitally after alveolar segmental osteotomy. J Maxillofac Surg. 1973; 1:85-91.

26. Banks P. Pulp changes after anterior mandibular subapical osteotomy in a primate model.J Oral Maxillofac Surg. 1977;5:39-48.

27. Sussman H., Moss S. Localized osteomyelitis secondary to endodontic-implant pathosis. A case report. J Periodontol 1993;64:306-10.

28. Margelos JT, Verdelis C. Irreversible pulpar damage of teeth adjacent to recently placed osseointegrated implants. J Endod 1995;21:479-82.

29. Piattelli A, Scarano A, Balleri P, Favero GA. Clinical and histologic evaluation of an active "implant periapical lesión". A case report. Int J Oral Maxillofac Implants 1998;13:713-6.

30. Laird B, Hermsen M, Gound T, Al Salleh F, Byarlay M, Vogt M. and cols. Incidence of endodontic implantitis and implant endodontitis occurring with single-tooth implants: a retrospective study. J Endod 2008;34:131624. 
31. Shabahang S. Effect of teeth with periradicular lesions on adjacent dental implants. Oral Surg Oral med Oral Pathol Oral Radiol Endod 2003;96:321-6.

32. Quirynen M, Vogels R, Alsaadi G, Naert I, Jacobs R, van Steenberghe D. Predisposing conditions for retrograde peri- implantitis, and treatment suggestions. Clin Oral Impl Res 2005; 16:599-08.

33. Siegenthaler D, Jung R, Hoderegger C, Roos M, Hammerle Ch. Replacement of teeth exhibiting periapical pathology by inmediate implants. A prospective, controlled clinical trial. Clin. Oral Impl Res. 2007; 18:72737.

34. Lindeboom $\mathrm{H}$. Inmediate placement of implants in periapical infected sites. A prospective randomized study in 50 patients. Oral Surg Oral Med Oral Pathol Oral Radiol Endod. 2006;101:705-10.

35. Crespi R, Cappare P, Gherlone E. Fresh-socket Implants in periapical infected sites in humans. J Periodontol 2010;81:378-83.

36 Chang SW, Shin SY, Hong JR, Yang SM, Yoo HM, Park DS. Inmediate implant placement into infected and no infected extraction sockets: a pilot study. Oral Surg Oral Med Oral Patol Oral Radiol Endod 2009;107:197-203.

37 Misch C.E, Misch-Dietsh F.,Sils J, Barboza E, Cianciola L, Kazor Ch. Posterior implant single-tooth replacement and status of adjacent teeth during a 10 year period: a retrospective report. J. Periodontol 2008;79:2378-82.

38. Brisman D, Brisman A, Moses M. Implant failures associated with asymptomatic endodontically treated teeth. J Am Dent Assoc. 2001 132:191-195.

39. Drago C.J. Letters. J Am Dent Assoc. 2001;132:577.

40. Hutter J.W. Letters. J Am Dent Assoc. 2001; 132: 853-5.

41. Steiner D. The resolution of a periradicular lesion involving an implant. J Endod. 2008; 34:330-5.

42. Tae-Ju Oh, Yoon J, Wang H. Management of the implant periapical lesion: a case report. Implant Dent 2003;12:41-6.

43. Tseng Ch, Chen Y, Pan I, Weber H. Peri-implant pathology caused by periapical lesion of an adjacent natural tooth: a case report. Int J Oral Maxillofac Implants 2005;20:632-5.
44. Chaffe N, LowdenK, Tiffee J, Cooper L. Periapical abscess formation and resolution adjacent to dental implants: a clinical report. J Prosthet Dent 2001;85:10912.

45. Romanos G. Implant periapical lesions: etiology and treatment options. J Oral Implantol 201 1;37:53-63.

46. Balshi S, Wolfinger G, Balshi T. A retrospective evaluation of a treatment protocol for dental implant periapical lesions: long-term results of 39 implant apicoectomies. Int J Oral Maxillofac Implants 2007;22:267-72.

47. Tehemar S. Factors Affecting heat generation during implant site preparation: a review of biologic observations and future considerations. Int J Oral Maxillofac Implants 1999;14:127-36.

48. Mohamed J., Shivakumar B, Sudarsan S, Arun K, Kumar T. Retrograde peri-implantitis. J Indian Soc Periodontol 2010;14:57-65.

49. Novaes A. Inmediate implants placed into infected sites: a clinical report. Int J Oral Maxillofac Implants 1995; 10 : 609-13.

50. Casap N, Zeltser CH, Wexler A, tarazi E, Zeltser R. Inmediate placement of dental implants into debrided infected dentoalveolar sockets. J Oral Maxillofac Surg. 2007;65:384-92.

51. Silva G, Oliveira D, Viera T, Magalhaes C, Moreira A. Unusual presentation of active implant periapical lesions: a report of two cases. J Oral Sci 2010;52:491-4.

52. Naves M., Horbylon BZ, Gomes Cde F, Menezes HH, Bataglion C, Magalhães D. Inmediate implants placed into infected sockets: a case report with 3 years follow up. Braz Dent J. 2009;20:254-8.

53. Abu-Rass M. Interim endodontic therapy for alveolar socket bone regeneration of infected hopeless teeth prior to implant therapy. J Oral Implantol 2010;36:3660.

\section{CORRESPONDENCIA}

Dr. Juan J. Segura-Egea

Dpto. Estomatología, Universidad de Sevilla

Avicena, $\mathrm{s} / \mathrm{n}$

41009 Sevilla

Correo electrónico: segurajj@us.es 